\title{
A study of amorphization energies in silicon for different implantation parameters
}

\section{E. Friedland ${ }^{\mathrm{a}}$}

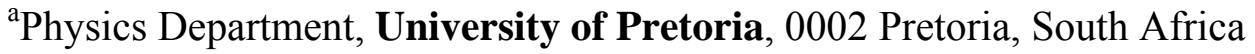

\begin{abstract}
Damage profiles were obtained from $\alpha$-particle channeling spectra of Si $(111)$ samples implanted with different fluences of $200 \mathrm{keV}$ carbon and $350 \mathrm{keV}$ argon ions at liquid nitrogen temperature. Critical damage energies were extracted by comparing the experimentally determined boundary positions of amorphized zones with elastic energy transfer densities obtained from TRIM simulations. A similar analysis was also done of previously published damage profiles of $2 \mathrm{MeV}$ self-ion implantations. Plotting the obtained amorphization energies as a function of inelastic energy densities transferred to the silicon lattice yield incompatible results for these three ion species. The reasons for the observed discrepancies are most probably erroneous silicon stopping powers employed by the TRIM code.
\end{abstract}

\section{Article Outline}

1. Introduction

2. Experimental method

3. Data analysis

4. Discussion and conclusion

Acknowledgements

References 


\section{Introduction}

At ion energies encountered in typical industrial applications damage accumulation in irradiated samples is thought to be exclusively a result of elastic atomic collisions. Direct defect creation by inelastic collisions at electronic stopping powers in the low $\mathrm{keV} / \mathrm{nm}$ range is not expected to contribute via the known mechanisms of either local melting [1] or Coulomb explosion [2]. However, this does not imply that inelastic processes cannot influence the damage efficiency of elastic collisions. Evidence for this effect was recently observed in a variety of semiconductors [3], [4] and [5]. It was proposed that electronic excitations impact on damage efficiency by two opposing mechanisms, namely radiation induced annealing and reduction of binding energies. Up to now results indicate that in mono-elemental semiconductors the first mechanism dominates, while in some binary materials the reduction of binding energy seems to be more important. However, the results for silicon are based on a re-analysis of measurements done about 20 years ago using an optical reflectivity method [6]. Although many ion implantation studies in silicon have been reported in the literature during the last two decades, most of them could not be used for determining amorphization energies. They were either not well enough documented to extract reliable data for this type of analysis or were performed at room temperature, where thermal effects, which are already discernible at $210 \mathrm{~K}$ [6], [7], [8] and [9], significantly modify defect concentration. Transition of crystalline to amorphous silicon is believed to be due to the formation of di-vacancies and diinterstitials during ion implantation, which rearrange to generate the typical five- and seven-member rings observed in the amorphous state [9] and [10]. However, this mechanism seems to be strongly dependent on substrate temperature during implantation. At high temperatures the preferred formation of extended defects leads to incomplete amorphization, while at liquid nitrogen temperature complete amorphization and a remarkable reduction of extended defects were observed [11].

In this work, damage profiles of carbon and argon implants are determined and the extracted dependence of amorphization energies on inelastic energy transfer densities are compared with the $2 \mathrm{MeV}$ self-ion implantation measurements [6] subjected to a similar analysis. 


\section{Experimental method}

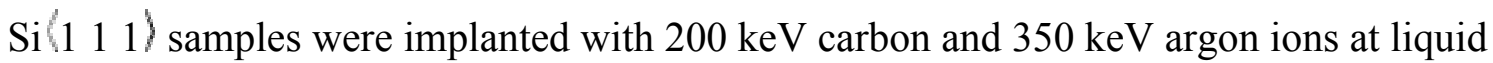
nitrogen temperature with ion fluences ranging from $1.5 \times 10^{14}$ to $10 \times 10^{14} \mathrm{~cm}^{-2}$. To prevent target heating dose rates were kept below $10^{13} \mathrm{~cm}^{-2} \mathrm{~s}^{-1}$. Furthermore targets were tilted $7^{\circ}$ relative to normal incidence to minimize channeling effects.

Damage profiles were investigated by $\alpha$-particle channeling at room temperature in a backscattering geometry employing a three-axes precision goniometer. The analyzing particle beam was collimated at a spot of $1 \mathrm{~mm}$ diameter and the current of approximately $10 \mathrm{nA}$ was measured directly on the target. A ring-shaped electrode in front of the target was kept at a negative potential of $300 \mathrm{~V}$ to suppress secondary electrons. Sufficient counting statistics was obtained by collecting an integrated charge of $4 \mu \mathrm{C}$. Backscattered particles were observed at $165^{\circ}$ by a surface barrier detector telescope with an acceptance angle of $2^{\circ}$. Aligned backscattering spectra were obtained at beam energies of 1.5 and $1.8 \mathrm{MeV}$. These channeling spectra were normalized to random spectra collected during rotation of the sample about an axis tilted by approximately $5^{\circ}$ relative to the channeling direction. Energies of the backscattered particles are converted to a depth scale by using the energy loss data of Ziegler [12]. Depth resolution near the surface is limited by the system's energy resolution of $12 \mathrm{keV}$ to approximately $24 \mathrm{~nm}$, which increases to $39 \mathrm{~nm}$ at a depth of $500 \mathrm{~nm}$ because of energy straggling.

\section{Data analysis}

Typical $\alpha$-particle channeling spectra for different carbon and argon fluences are shown in Fig. 1 together with random spectra. The regions where the count rate of the aligned spectra equals that of the random yield correspond to defect densities, where the backscattering probability is similar to that of a completely disordered region. In channeling analysis this is generally considered as 'amorphous', although strictly speaking this might not necessarily be the case. However, for the following discussion this uncertainty is immaterial, as only boundary positions of the regions are compared, where the backscattering yield approaches the random level. It is therefore independent of a particular scattering model and only assumes that defect densities are the same at all 
positions where the normalized backscattering yield just reaches unity. By obtaining the corresponding depth dependent elastic and inelastic energy densities at these positions, critical damage energies as a function of the inelastic energy densities are extracted.
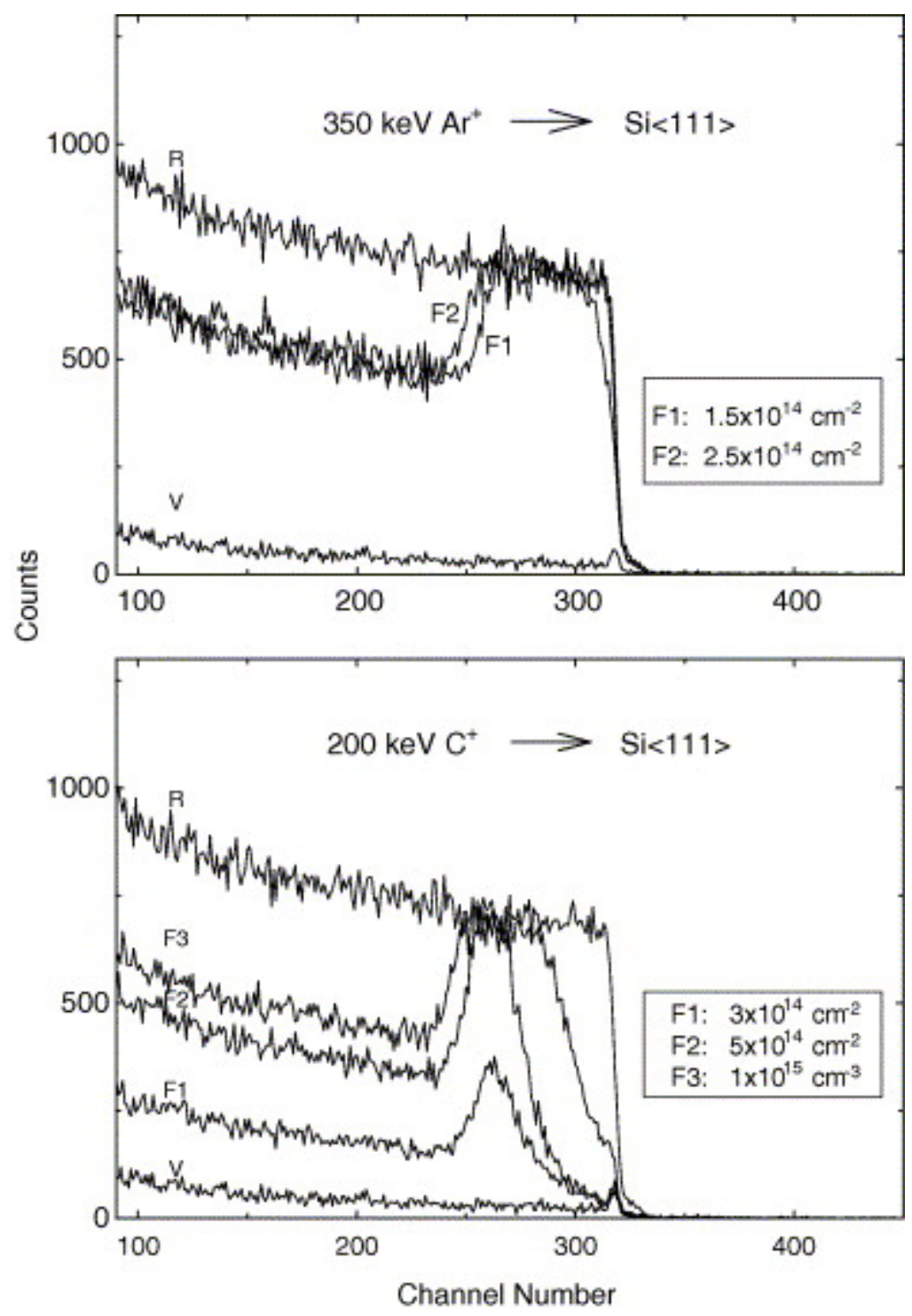

Fig. 1. Aligned backscattering spectra before (V) and after implantation of carbon and argon ions into silicon at $T \sim 80 \mathrm{~K}$ with different fluences obtained with $1.8 \mathrm{MeV} \alpha$ particles at a scattering angle of $165^{\circ}$. Also shown are random spectra (R).

For carbon bombardment the amorphization fluence is approximately $5 \times 10^{14} \mathrm{~cm}^{-2}$, leading to a thin amorphized layer at $(392 \pm 36) \mathrm{nm}$. For argon ions the critical fluence is probably near $10^{14} \mathrm{~cm}^{-2}$. At higher fluence buried amorphized zones occur, which at still 
higher values will eventually reach the surface. For the carbon implant with a fluence of $10^{15} \mathrm{~cm}^{-2}$, zone boundaries are found at depths of $(243 \pm 32) \mathrm{nm}$ and $(480 \pm 38) \mathrm{nm}$ below the surface. The argon implant at a fluence of $1.5 \times 10^{14} \mathrm{~cm}^{-2}$ produces an amorphized zone from $(93 \pm 27)$ to $(392 \pm 36) \mathrm{nm}$, while after implanting a fluence of $2.5 \times 10^{14} \mathrm{Ar}^{+} \mathrm{cm}^{-2}$ the amorphized region extends from approximately the surface up to a depth of $(444 \pm 37) \mathrm{nm}$. Bohr straggling is assumed for estimation of the quoted errors. For the calculation of damage energies one must bear in mind, that elastic energy transfer to the lattice is significantly less than the ion's nuclear stopping power. The latter is the energy transferred during elastic ion-atom collisions, leading to the displacement of primary knock-on atoms and phonon excitations. An appreciable fraction of this energy is subsequently lost by inelastic collisions of the primary and secondary recoil atoms and must therefore be added to the ion's electronic stopping power to obtain the total inelastic energy deposition density as a function of penetration depth. Fig. 2 shows the results obtained from TRIM simulations [13] for elastic and inelastic energy transfer densities to the silicon lattice for carbon, argon and silicon ions at energies used in this study and that of [6]. Superposed are bar diagrams showing typical depth distributions of collision cascades for a single ion. 

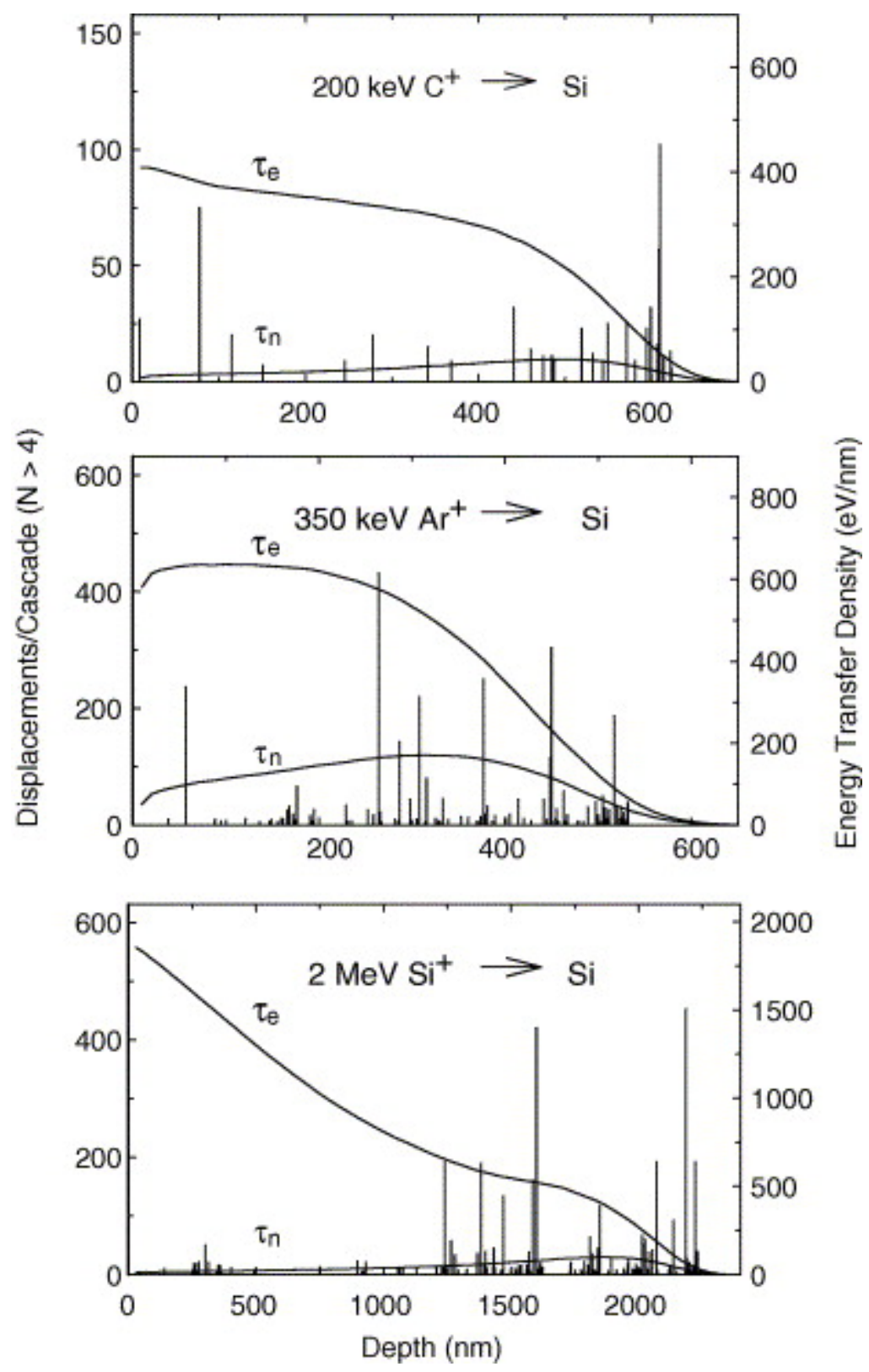

Fig. 2. Total elastic $\left(\tau_{\mathrm{n}}\right)$ and inelastic $\left(\tau_{\mathrm{e}}\right)$ energy densities transferred to a silicon lattice during the stopping of $200 \mathrm{keV}$ carbon, $350 \mathrm{keV}$ argon and $2 \mathrm{MeV}$ silicon ions. Also shown as a bar diagram are typical distributions of collision cascades $(N>4)$ caused by a single ion. The data were calculated using the TRIM 98 code [13].

Damage energy $\varepsilon(x)$ at depth $x$ is defined as

$$
\varepsilon(x)=F \tau_{\mathrm{n}}(x) / \rho,
$$


with $F$ the fluence, $\tau_{\mathrm{n}}(x)$ the elastic energy deposition density at depth $x$ and $\rho$ the atomic density of the substrate. From this the amorphization energies at the boundary positions are calculated and plotted as a function of the inelastic energy transfer density $\tau_{\mathrm{e}}$ in Fig. 3 . Included in this plot are the results obtained from an analysis of published damage profiles of $2 \mathrm{MeV}$ silicon implants at $125 \mathrm{~K}$ by optical reflectivity depth profiling [6], for which a similar cautionary comment on the meaning of the term 'amorphous' is relevant. According to these results a critical fluence of about $2.5 \times 10^{14} \mathrm{Si}^{+} \mathrm{cm}^{-2}$ produces a thin amorphous layer at the maximum of the elastic energy distribution, which occurs at $1.86 \mu \mathrm{m}$. A fluence of $4.5 \times 10^{14} \mathrm{Si}^{+} \mathrm{cm}^{-2}$ resulted in an amorphous region ranging from about $1.52-2.22 \mu \mathrm{m}$, while amorphization from a depth of 0.79 to approximately $2.36 \mu \mathrm{m}$ was obtained with a fluence of $12 \times 10^{14} \mathrm{Si}^{+} \mathrm{cm}^{-2}$. Assuming that self-ion implantation into silicon is causing no serious change in reflectivity, depth dependent amorphization energies were calculated and also plotted in Fig. 3 as a function of the inelastic energy transfer. Errors were estimated by assuming a depth uncertainty of $20 \mathrm{~nm}$.

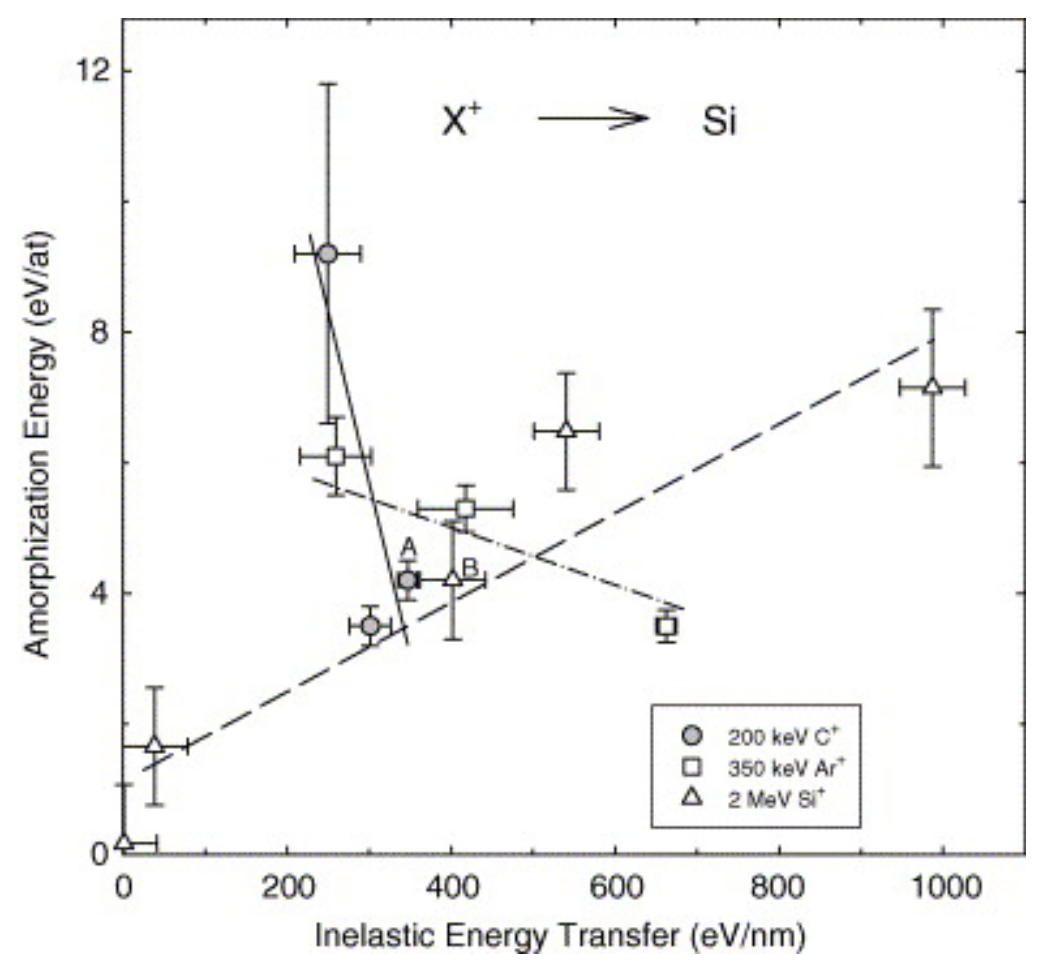

Fig. 3. Amorphization energies for silicon for $200 \mathrm{keV}$ carbon and $350 \mathrm{keV}$ argon implants with different fluences at $T \sim 80 \mathrm{~K}$. Also shown are amorphization energies for $2 \mathrm{MeV}$ silicon ions extracted from results reported by Hecking et al. [6]. Data were 
obtained by comparing experimentally determined boundary positions of amorphized zones with elastic energy transfer densities at these positions computed by TRIM 98 simulations [11]. Straight lines are drawn to guide the eye.

\section{Discussion and conclusion}

If defect densities in any particular material would be determined by the deposited elastic energy density only, the boundaries of amorphized zones would occur at identical damage energies and would furthermore be independent of ion energies and species. It would also not depend on penetration depth or any other parameter linked to it like the inelastic energy transfer density. This assumption of constant damage efficiency is, of course, unrealistic. It completely ignores any transient effects on defect dynamics and atomic binding energies by electron-phonon interactions occurring simultaneously during atomic collisions in its immediate neighborhood. In the vicinity of an ion track electronic stopping momentarily creates a high density of excited atoms embedded in a hot electron gas. In this environment lattice binding energy is reduced, while simultaneously the possibility of point defect annealing increases. Depending on which of these two processes dominate, damage efficiency is either enhanced or reduced. The magnitude of this effect depends on the velocities and effective charges of the colliding ion and recoiling atoms and hence is directly correlated with the depth dependent inelastic energy density transferred to the lattice. Plotting amorphization energies as a function of inelastic energy density should therefore not depend on ion specie and energy, which is in total disagreement with the results displayed in Fig. 3. One might argue that this is due to point defect recombination and clustering in high density collision cascades, which is another mechanism influencing damage efficiency and should occur with increasing probability in regions where correlated collision cascades overlap. It is expected that such non-linear processes play a dominant role near the end of the ion's range, particularly with heavier ions. A closer look, however, reveals that this cannot possibly explain the peculiar data shown in Fig. 3. For example the amorphization energies obtained from the carbon implant at a depth of $243 \mathrm{~nm}$ (data point A) and from the silicon implantation at $2220 \mathrm{~nm}$ (data point B) are nearly identical, although the first one was obtained from a region of low and the latter from a region of high cascade density (see Fig. 2). 
To interpret the apparent disagreement between the plotted dependencies on inelastic energy density, one has to be aware of the fact that the determination of amorphization energies is indirect in nature. The above analysis depends critically on the knowledge of accurate elastic and inelastic energy densities transferred to the target material as a function of penetration depth. In all cases these quantities were calculated from stopping powers obtained from TRIM simulations [13], which claim an average accuracy of about $5 \%$ in fair agreement with most experimental investigations. However, a notable exception was reported for $150 \mathrm{keV}{ }^{13} \mathrm{C}$ ions into silicon [14], where TRIM estimates a projected range of $R_{\mathrm{p}} \approx 466 \mathrm{~nm}$, which is approximately $15 \%$ larger than experimentally observed. Furthermore a strongly asymmetric implantation profile with negative skewness is predicted, while experimentally a Gaussian distribution is observed. Obviously stopping powers adopted by TRIM for this particular ion and energy are too small and the question arises, whether this is also the case for other implantations into silicon, which could explain the drastic inconsistencies observed in Fig. 3.

In Fig. 4, the experimentally determined positions of amorphized zones are superposed on the elastic energy transfer densities obtained from TRIM simulations. Striking differences regarding the positions of these zones relative to the maximum of the elastic energy density are revealed for the three ion species. In the case of the carbon implant, the amorphized zone does not even include the peak of the energy density distribution, which is the reason for the steep negative slope shown in Fig. 3 for this ion. If a similar under-estimation of stopping powers as observed by [14] is assumed for this implantation, the energy density peak would shift into the amorphized region and would deliver results consistent with those obtained for the argon implants. However, it would still be in disagreement with the results from the high-energy silicon implants. The latter results seem more likely to be correct, as extrapolation to zero inelastic energy density leads to an amorphization energy of approximately $1 \mathrm{eV} /$ atom, which agrees quite well with what is expected if the average dissociation energy of a di-vacancy and a diinterstitial is subtracted from the lattice binding energy. 


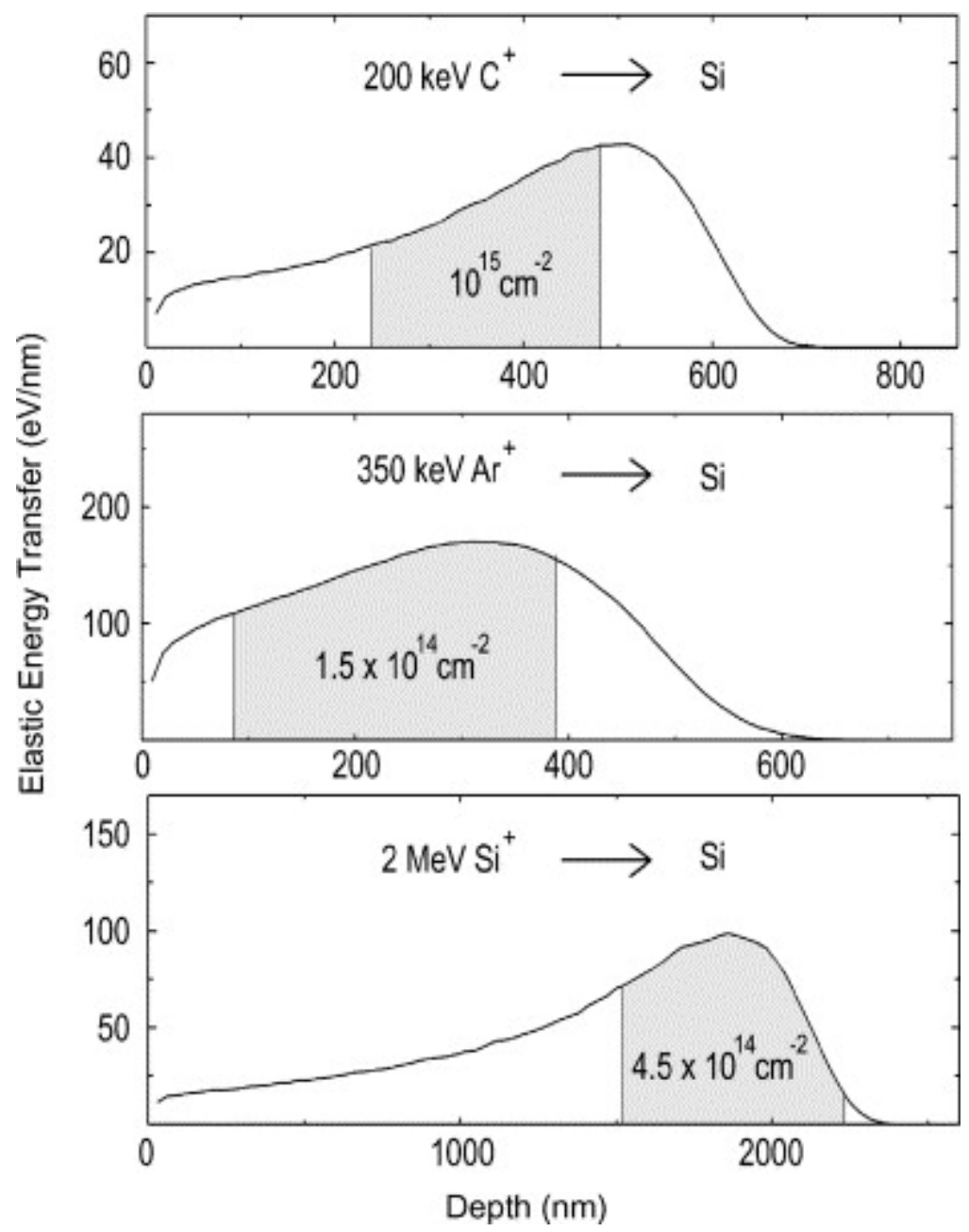

Fig. 4. Experimentally determined amorphized regions in silicon for different implantation parameters compared with elastic energy transfer densities obtained from TRIM 98 simulations [13].

In Fig. 5, the electronic energy loss data used in TRIM 98 are compared with those of the corresponding universal stopping power formula proposed by the Heidelberg group [15], which was empirically obtained from data at $E>10 \mathrm{keV} / \mathrm{amu}$. Although these estimates are only validated for energies above $120 \mathrm{keV}$ for carbon, $280 \mathrm{keV}$ for silicon and $400 \mathrm{keV}$ for argon ions, a comparison might be instructive. Firstly, from Fig. 5(d) it follows that the experimentally observed amorphous regions would in all cases be shifted to approximately $10 \%$ larger depths than shown in Fig. 4 . On the other hand the smaller electronic stopping powers for the implants (Fig. 5(a)-(c)) will also shift the elastic and inelastic transfer distributions to larger depths. One might therefore be tempted to argue, that nothing fundamentally will change by using the Heidelberg data. However, this is 
probably a too simplistic argument. The employed analysis depends only partly on the position of the amorphized zone relative to the corresponding elastic energy transfer curve and is furthermore strongly depended on the shape of the latter distribution. This shape depends sensitively on the energy distributions of the recoiling target atoms along the track of the ion, which are not linear functions of the total stopping power. It will also depend on the ratio of inelastic and elastic stopping contributions, which differ significantly for the three ion species. To get a clear picture of the influence of modified stopping powers on amorphization energies, they have to be incorporated into the TRIM code. This, however, seems not to be an appropriate method, as the main contribution to the damage energy stems from low energy collisions, where the Heidelberg data are not necessarily valid.

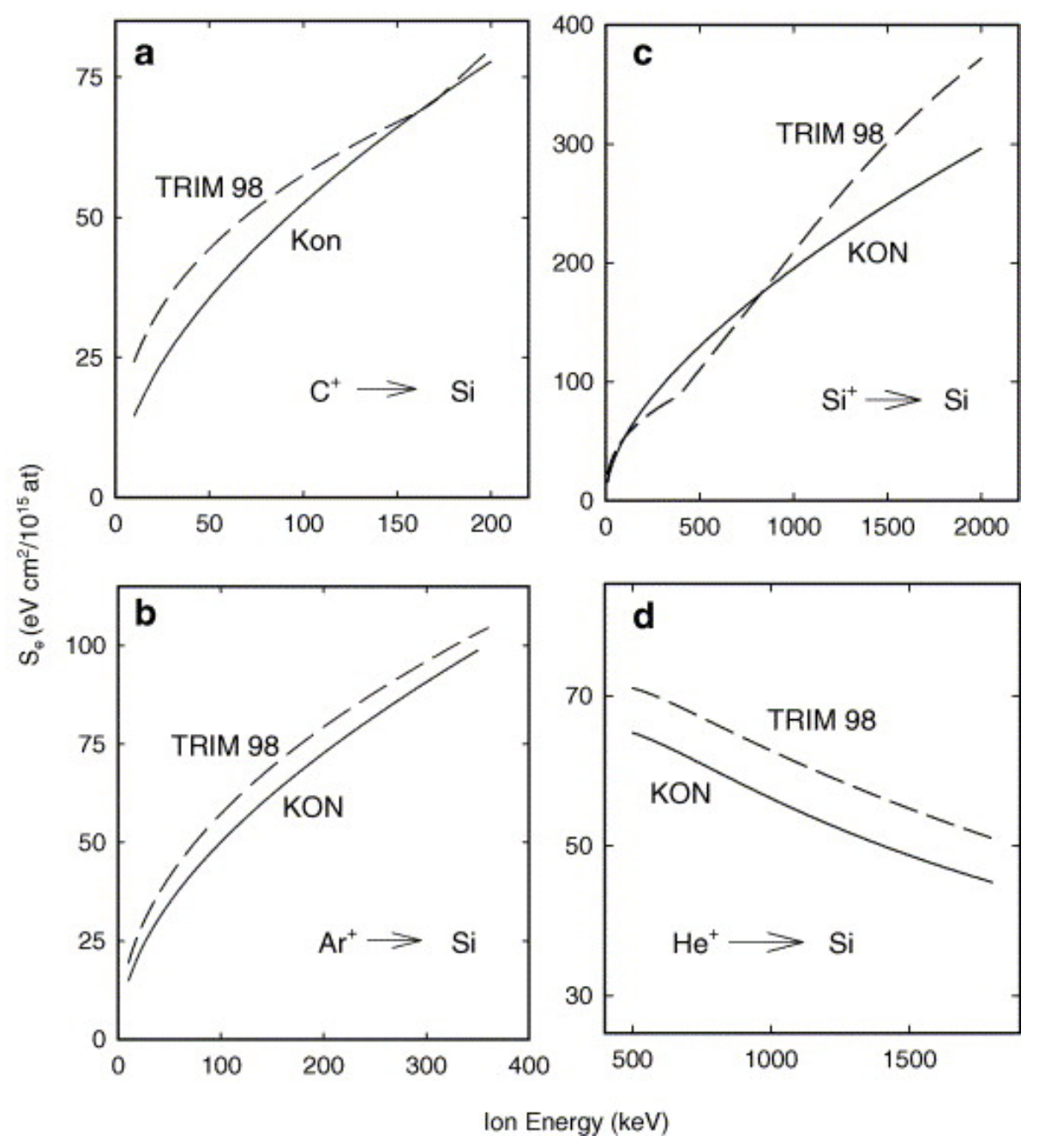


Fig. 5. Comparison of inelastic stopping powers in silicon employed by TRIM [13] and the universal electronic stopping power formula of [15] for carbon (a), argon (b), silicon (c) and helium ions (d) at appropriate energy regions.

A possible reason for the conflicting results obtained in this study might be due to the influence of silicon's band-gap, which is assumed to reduce electronic stopping at low energies. To take this effect into account, TRIM assumes for narrow band-gap semiconductors at energies below the Fermi level a decreasing stopping power according to $S_{\mathrm{e}} \quad v^{0.7}$ instead of the usual linear velocity dependence. This might not be appropriate for highly disordered silicon considered in this study.

At this stage no conclusion can therefore be drawn on whether or not damage efficiency is reduced in silicon by electronic stopping as is observed in the other two monoelemental semiconductors diamond and germanium. To finally settle this question, more reliable stopping powers in silicon than currently available are needed.

\section{References}

[1] C. Dufour, E. Paumier and M. Toulemonde, Radiat. Eff. Defect. Solids 126 (1993), p. 19.

[2] A. Dunlop, D. Lesueur, J. Morillo, J. Dural, R. Spohr and J. Vetter, Nucl. Instr. and Meth. B 48 (1990), p. 419.

[3] E. Friedland, Nucl. Instr. and Meth. B 217 (2004), p. 396.

[4] E. Friedland, H.D. Carstanjen, G. Myburg and M.A. Nasr, Nucl. Instr. and Meth. B 230 (2005), p. 129.

[5] E. Friedland, Surf. Coat. Technol., in press.

[6] N. Hecking, K.F. Heidemann and E. te Kaat, Nucl. Instr. and Meth. B 15 (1986), p. 760 .

[7] J.K.N. Lindner, R. Zuschlag and E.H. Te Kaat, Nucl. Instr. and Meth. B 62 (1992), p. 314.

[8] F.F. Morehead and B.L. Crowder, Radiat. Eff. 6 (1970), p. 27.

[9] L. Pelaz, L.A. Marques and J. Barbolla, J. Appl. Phys. 96 (2004), p. 5947.

[10] T. Motooka, Thin Solid Films 272 (1996), p. 235. 
[11] T. Suzuki, H. Yamaguchi, S. Ohzono, N. Natsuaki, in: Extended Abstract of the 22nd International Conference on Solid State Devices and Materials, 1990, p. 1163. [12] J.F. Ziegler, The Stopping and Ranges of Ions in Matter Vol. 4, Pergamon, New York (1977).

[13] J.F. Ziegler, J.P. Biersack and U. Littmark, The Stopping and Ranges of Ions in Matter Vol. 1, Pergamon, New York (1985).

[14] E. Friedland, M. Hayes, S. Kalbitzer and P. Oberschachtsiek, Nucl. Instr. and Meth. B 85 (1994), p. 272.

[15] G. Konac, Ch. Klatt and S. Kalbitzer, Nucl. Instr. and Meth. B 146 (1998), p. 106.

Tel.: +27 12 4202453; fax: +27 123625288 . 